\author{
Anita Staroń \\ Université de Łódź, Pologne \\ http://dx.doi.org/10.18778/8088-896-8.15
}

\title{
RACHILDE, HOMME DE LETTRES SEXE ET EXCLUSION
}

À une époque où la voix des féministes se fait plus forte et où le nombre d'auteurs femmes va croissant, la position de Rachilde (née en 1860, débutant à Paris en 1880) paraît ambiguë, sinon paradoxale. Femme écrivain elle-même, auteure de plusieurs romans prisés par le public, critique littéraire au Mercure de France, elle pourrait devenir figure de proue du mouvement féministe. Et pourtant, elle bafoue systématiquement ses revendications ; elle reste aussi très critique envers la littérature de ses consœurs, dont elle refuse de faire partie. Dès lors, il semble intéressant de chercher les causes d'une telle attitude. L'étendue du sujet et, plus encore, les déclarations contradictoires de Rachilde elle-même, nous permettront tout au plus, dans ce texte, de formuler quelques hypothèses. Nous tâcherons par contre de creuser davantage le problème de l'appartenance de Rachilde à une - ou plusieurs - minorité(s).

\section{Parcours biographique}

Dès sa naissance, en 1860, Marguerite Eymery est confrontée à plusieurs handicaps qui, s'ils aident à former son caractère indépendant, lui enseignent également la solitude et le sentiment d'être une exception. Née avec une jambe plus courte, la condamnant à boiter, elle ne peut compter sur un support affectueux de sa mère ; au contraire, celle-ci lui fait la vie dure, en l'élevant dans une discipline sévère sous prétexte de faire d'elle « une jeune fille comme il faut ». Rachilde en garde avant tout une conviction qui l'accompagnera pendant toute sa vie : la femme est incomparablement plus limitée dans ses actions que l'homme ${ }^{1}$. Sentiment renforcé par l'observation de son père, sévère lui aussi, distant, et de toute évidence mécontent d'avoir une fille au lieu d'un garçon. Il s'en console, donnant à Marguerite une éducation martiale :

Puisque le malheur voulait que je fusse une fille, on m'avait formé le caractère en me faisant risquer tous les dangers que l'on cherche généralement à éviter aux

\footnotetext{
1 Rachilde, Quand j'étais jeune, Paris, Mercure de France, 1947.
} 
êtres faibles. Je savais monter à cheval, faire des armes, sauter des barrières d'un mètre et suivre les grandes chasses au galop... [...] Mon père $[\ldots]$ n'appréciait que le courage et n'accordait d'attention à ce garçon manqué que lui représentait sa fille, que lorsqu'il le sentait capable d'exécuter un ordre ou de respecter une consigne ! ${ }^{2}$

D’autre part, sa mère, déçue par le mariage et détestant, par conséquent, tous les hommes, commence à compléter l'éducation traditionnelle par un enseignement pseudo-féministe qui vise surtout le manque « de toute obéissance vis-à-vis de cet homme parce que l'homme : «animal immonde et égoïste» n'a pas le droit d'exercer sa détestable influence sur les gens vertueux $\gg^{3}$. Cela n'empêche pas la jeune fille d'admirer son père, malgré, ou peut-être à cause de son éloignement.

Toutes ces contrariétés sont sans doute à l'origine de son goût - ou faudrait-il parler de nécessité - de se cacher derrière des masques :

On m'avait appris à vaincre la peur, à ne jamais avouer mon impuissance à réaliser un tour de force, malgré mon dégoût de toute entreprise un peu hasardeuse, et il me fallait, bon gré, mal gré, essayer de me dépasser moi-même, ce qui ne tendait guère qu'à devenir, tout simplement, un bon acteur sachant jouer son rôle ${ }^{4}$.

On ne saurait non plus exclure leur influence sur la façon dont Rachilde abordera la question de l'identité sexuelle.

\section{Rapport ambigu au sexe}

L'écriture devient pour Marguerite une autre manière d'échapper à la réalité décevante - et une véritable vocation. Elle débute dans des journaux de sa province périgourdine $e^{5}$, signant d'un pseudonyme trouvé dans ses lectures : Rachilde serait le nom d'un gentilhomme suédois du XVI siècle. De ce temps, elle rapporte une anecdote révélatrice : lorsqu'elle parlait au directeur du journal habillée d'une manière habituelle pour son sexe et état, il était condescendant et ne discutait pas de questions de métier ; mais quand elle arrivait en son costume d'homme qu'elle mettait pour aller à cheval, le directeur changeait complètement d'attitude et lui montrait beaucoup plus de considération. Elle en est venue à se demander s'il ne croyait pas à l'existence de deux personnes distinctes, une

2 Ibidem, p. 49.

3 Rachilde, Pourquoi je ne suis pas féministe, Paris, Éditions de France, 1928, coll. « leurs raisons » dirigée par André Billy, p. 16-17.

4 Rachilde, Quand j'étais jeune..., p. 49.

5 Entre autres pour L'Écho de la Dordogne. 
sœur et un frère ${ }^{6}$. Cette expérience, liée au mépris pour le sexe féminin, affiché par son père, put contribuer à sa décision de s' habiller en homme et de couper les cheveux court après son arrivée à Paris. La jeune femme prétextait la commodité et le coût infiniment plus bas d'une toilette masculine, mais ses cartes de visite témoignent d'une prise de position beaucoup plus ferme : « Rachilde homme de lettres $\gg$.

Devenue critique au Mercure de France, elle cultive cette ambiguïé. Dans ses comptes rendus, elle mélange les genres masculin et féminin quand elle parle à la première personne :

Je n'aime pas du tout ces œuvres-là, mais quand, par hasard, malgré mon ennui à les lire, je vois éclater l'effort du travailleur qui l'a voulue aussi belle et bonne que possible, je suis content et je ne sais pas railler des goûts qui ne sont pas les miens. [...] ...vers la fin, malgré quelques phrases déclamatoires, je me suis attendri sur le sort de Louis Léclat... ${ }^{7}$

L'amour mâle domine à cause de l'usage... du temps ou des préférences du romancier, qui m'a l'air de sacrifier volontiers la femme à l'éphèbe, ce dont je le félicite, probablement parce que je suis une femme ! ${ }^{8}$

Je suis étonnée qu'un homme ose l'offrir telle qu'elle lui est apparue. [...] Je suis satisfait de sentir un auteur, volontairement ou non, côtoyer ce joli précipice?

Il semblerait que le genre utilisé s'adapte à l'intention du moment : lorsque Rachilde veut souligner son autorité de critique, elle choisira la forme masculine ; mais pour les besoins d'une discussion thématique, où une opposition entre les sexes lui parait de mise, elle n’hésite pas à se réclamer de sa condition féminine.

\section{Rachilde et le féminisme}

Dès lors, il se pose la question du rapport de l'écrivaine au mouvement féministe qui se développe précisément à son époque. Son indépendance, la position qu'elle a su se créer dans le monde littéraire pourraient faire d'elle une adhérente de valeur. Or, il n'en est rien. Si elle mentionne souvent le mouvement ou ses adeptes, c'est toujours pour les bafouer. On en trouve de nombreux exemples dans ses comptes rendus des années 1890-1910. Elle parle du « froncement de

${ }^{6}$ Rachilde, Quand j'étais jeune..., p. 56-57.

7 Rachilde, C. R. de L. Lumet, La Fièvre, Mercure de France juillet 1898, p. 230. Tous les comptes rendus de Rachilde cités ici ont paru au Mercure de France.

8 C. R. d'A. Delacour, Le Pape rouge, novembre 1901, p. 493-494.

9 C. R. de P. Valdagne, L'Amour par principes, octobre 1898, p. 203. 
narines que l'on a toujours en pensant aux émancipatrices de l'ère nouvelle $\gg$ et déclare que «le féminisme, qui tend à vouloir plus que ce que la nature nous donne, tend à séparer de plus en plus les sexes et, surtout, à en ridiculiser un aux dépens de l'autre $\gg{ }^{10}$. Reprenant pour son compte les arguments éternels des antiféministes, elle souscrit à l'idée de la différence intellectuelle entre les sexes. Ainsi, elle loue le Dictionnaire de la Femme, par G. Cerfberr et M.V. Ramin, à cause de sa simplicité, propre à satisfaire les femmes que des dictionnaires ordinaires, « très compliqué[s] et trop scientifique $[\mathrm{s}] \gg$ effraient $^{11}$. Car les femmes, selon Rachilde, ont l'esprit borné par rapport au cerveau de l'homme, « plus vaste, ou fonctionnant mieux plus longtemps $\gg^{12}$. Elles sont, observe-t-elle, limitées aussi par leur physique : elles vieillissent, et elles en subissent les conséquences autrement que les hommes ${ }^{13}$. En somme, les femmes devraient garder leur place traditionnelle au ménage, qui leur convient mieux, même si elles n'en sont pas toujours conscientes - tel est le sens de sa réponse à l'Enquête sur le féminisme menée en $1902^{14}$.

Encore en 1928, elle explique sur plusieurs pages Pourquoi [elle n'est] pas féministe ${ }^{15}$. Elle y reprend l'argument de la faiblesse intellectuelle de la femme : « il y a des choses qu'elle ne comprend pas, qu'elle ne comprendra jamais. Et est-ce bien utile qu'elle les comprenne ? » et n’hésite pas à abaisser ses consœurs :

Les femmes sont les frères inférieurs de l'homme, simplement parce qu'elles ont des misères physiques les éloignant de la suite dans les idées que peuvent concevoir tous les hommes en général, même les moins intelligents. [...] Répandre l'instruction sur les simples d'esprit, c'est répandre tous les maux de la boîte de Pandore ${ }^{16}$.

Avant de chercher d'autres raisons de cette réfutation du féminisme, on pourrait invoquer les expériences particulières de Rachilde, fille d'une femme instable, névrosée, enfin malade psychique ${ }^{17}$; cela permettrait d'expliquer sa méfiance de l'«éternel féminin », qu'elle avait toutes les raisons d'associer à sa mère : « je continue à regarder comme un danger tout accaparement cérébral de la femme parce que son cerveau est peut-être moins solide que celui de l'homme $\gg$, déclare-t-elle ${ }^{18}$.

10 C. R. de J. Leroy, Le Plaisir d'aimer, février 1904, p. 474.

11 Rachilde, Notice bibliographique du Dictionnaire de la Femme, Mercure de France, juillet 1897, p. 276.

12 C. R. de R. Behaine, La Conquête de la vie, septembre 1899, p. 778.

13 C. R. de C. Albane, L'Amour tout simple, janvier 1900, p. 191.

14 Le Carnet (ancien Carnet historique et littéraire), Tome XI, janvier-février-mars 1902 , p. 262-263.

15 Rachilde, Pourquoi je ne suis pas féministe...

16 Ibidem, p. 10-12.

17 Elle a passé les dernières années de sa vie dans un hôpital psychiatrique.

18 Rachilde, Pourquoi je ne suis pas féministe..., p. 21. 
Mais il semble que la raison principale de son incompréhension de la lutte des féministes se situe dans la position ambiguë par rapport à l'identité sexuelle, évoquée auparavant. En effet, lorsqu' elle aborde les problèmes de métier littéraire, elle se place en dehors de la catégorie féminine : « Il ne faut pas chercher dans les livres des femmes, voudraient-elles écrire comme des hommes, autre chose que leur âme, et il ne faut pas voir, en leur âme, autre chose que l'amour. [...] elles n'écrivent jamais que leur âme, sans réaliser d'autres âmes plus curieuses $\gg{ }^{19}$, proclame cette auteure d'ouvrages scandaleux qui se défendait, jusqu'à intenter des procès pour diffamation ${ }^{20}$, contre les rapprochements entre elle et les personnages de ses romans (on y reviendra). Selon toute probabilité, elle exprime cette opinion de la position «masculine » qu'elle prenait pour écrire. Elle avoue d'ailleurs ne pas appartenir à « la race des femelles, seules créatures vraiment indispensables à la vie normale, ni [à] la race des courtisanes qui sont également nécessaires à l'existence d'une société... puisqu'elles en sont le plus bel ornement $\gg$, et de « demeurer en reporter, c'est-à-dire de rester neutre en prenant des notes sans prendre parti $\gg^{21}$.

Or, si cette dernière déclaration est sujette à caution, puisqu'il semble que Rachilde veut, au contraire, prendre une position, il est clair aussi que, dans sa vie d'écrivain(e), surtout pendant les premières années de sa création, elle a dû se défendre contre des jugements qui se référaient à son sexe. Elle fut particulièrement sensible aux opinions formulées par la critique.

\section{Rachilde aux yeux de la critique}

Rachilde éveille, dans la fratrie critique, des émotions fortes. Ses œuvres fantasques, excentriques, jouant fréquemment sur une note pathétique, provoquent des commentaires allant d'un extrême à un autre, d'une outrance profonde à une admiration sans bornes. Mais Rachilde elle-même intrigue aussi et cela, dans une mesure beaucoup plus grande que si elle était un jeune homme. Ainsi, dès le début de sa carrière, elle est confrontée au nombre de commentaires qui visent son sexe et recourent aux stéréotypes de féminité. Il semble cependant qu'on peut y distinguer deux phases : celle où Rachilde est associée à d'autres femmes écrivains, et celle où, au contraire, elle en est séparée. Mais à chaque fois, son sexe est pris en compte.

${ }_{19}$ Comptes rendus de J. Laurenty, Joie Morte et de M. Lépine, Le Jour Prédit, mai 1897, p. 371.

${ }^{20}$ C'est ce qu'elle décrit dans sa préface du roman À mort, [in] Rachilde - Maurice Barrès. Correspondance inédite 1885-1914, édition établie et présentée par M. R. Finn, Brest, Centre d'Étude des Correspondances et Journaux Intimes/Faculté des Lettres/ C.N.R.S, 2002, p. 157-174.

${ }^{21}$ Rachilde, Pourquoi je ne suis pas féministe..., p. 84. 
Il sera peut-être utile de rappeler ici les réactions des contemporains au nombre croissant d'écrivains femmes. On saisit bien le ton général à partir de deux échantillons, parus à dix ans de distance : Les Bas-bleus de Jules Barbey d'Aurevilly (1878) et Le Massacre des Amazones de Han Ryner (1888). Nombre de femmes écrivains s'y trouvent fortement critiquées, surtout pour leurs aspirations à égaler les hommes. Tout au plus, observe Barbey, la femme pourra imiter l'homme sans jamais atteindre son talent mâle :

Les femmes peuvent être et ont été des poètes, des écrivains et des artistes, dans toutes les civilisations, mais elles ont été des poètes femmes, des écrivains femmes, des artistes femmes. [...] Mais quand elles ont le plus de talent, les facultés mâles leur manquent aussi radicalement que l'organisme d'Hercule à la Vénus de Milo [...]. Ainsi elles n'ont ni l'invention qui crée ou découvre, ni la généralisation qui synthétise, ni la force sans convulsion, car la force convulsive, passionnée, elles peuvent l'avoir en leur qualité de femmes $[\ldots]^{22}$.

Dix ans plus tard, Han Ryner ne voit pas le problème autrement : le bas-bleu est pour lui méprisable, car

Il n'écrit pas des livres de femme. Amante ou catin, il s'y refuse. Il est l'orgueilleuse amazone à qui il faut des victoires et des maîtresses. Apparente androgyne qui repousse son rôle naturel et, naïvement ou perversement, fait l'homme. Ange inepte qui se trompe, ou succube inquiet qui veut à son tour être l'incube. Ce qui constitue le bas-bleu ou amazone, c'est qu'un léger développement de ce qui semble viril en elle lui fait croire qu' intellectuellement elle est un homme ${ }^{23}$.

Rachilde ne figure pas, et pour cause ${ }^{24}$, dans l'ouvrage de Barbey ; et elle n'est pas trop «massacrée » par Han Ryner. D’autres critiques cependant n’hésitent pas à présenter la bizarrerie et l'excentricité de ses romans comme le résultat de son instabilité psychique, due à son sexe. À la parution de Monsieur Vénus, Henri Fouquier alias Colombine voit dans l'ouvrage une « curiosité de notre temps » emblématique du « détraquage » en littérature, et dans son auteur, une « folle » et une $\ll$ hystérique $»^{25}$. Parmi d'autres attaques, citons celle, particulièrement

${ }^{22}$ J. Barbey d'Aurevilly, Les Bas bleus, Paris, Société Générale de Librairie Catholique, 1878, p. XXII.

${ }_{23}$ Han Ryner, Le Massacre des Amazones, Paris, Chamuel, 1888, p. 4-5.

${ }^{24}$ Son début littéraire, Monsieur de la Nouveauté, voit le jour deux ans après la publication du livre d'Aurevilly.

${ }^{25}$ Colombine, compte rendu de Monsieur Vénus, Gil Blas, 29 septembre 1884. Faute de place, contentons-nous de rappeler à quel point, à cette époque, l'hystérie était perçue comme une maladie exclusivement féminine. C'est ainsi que l'avaient présentée Briquet, Huchard, Legrand du Saulle. Cf. à ce point G. Wajeman, « Psyché de la femme : note sur 
grotesque, du Dr Luiz ${ }^{26}$, qui après avoir décrit la romancière comme « une fleur fanée, une enfant pâle, fluette, sans jeunesse », arborant « une frimousse de gamin vicieux », passait à la présentation de son art :

Cette écrivassière pitoyable vit misérablement du produit de ses élucubrations spasmodiques. $[\ldots]$

Le livre de cet être dévoyé, d'un sexe incertain, a pour titre : Monsieur Vénus.

Le piquant du volume, pour les adeptes, consiste dans ce fait : d'une femme devenue par amour la fellatrix d'un pignouf!

Maurice Barrès, sans tomber dans de tels extrêmes, n'en présentait pas moins Monsieur Vénus comme « Les Liaisons dangereuses d'un bas-bleu » qu'il comparait à un monstre ${ }^{27}$. Dans sa préface à l'édition française de Monsieur Vé$n u s^{28}$, il refusait tout simplement à l'auteur tout pouvoir de réflexion sur son œuvre :

Les jeunes filles nous paraissent une chose très compliquée, parce que nous ne pouvons nous rendre compte qu'elles sont gouvernées uniquement par l'instinct, étant de petits animaux sournois, égoïstes et ardents. Rachilde, à vingt an $\mathrm{s}^{29}$, pour écrire un livre qui fait rêver un peu tout le monde, n’a guère réfléchi; elle a écrit tout au trot de sa plume, suivant son instinct.

Affirmant que, « dans toute son œuvre [...], Rachilde n'a guère fait que se raconter soi-même $\gg$, Barrès retrouvait un autre stéréotype de l'écriture féminine, à savoir l'impossibilité de faire abstraction de son propre tempérament et

l'hystérique au XIX' siècle », Romantisme, 1976, n¹3-14. Mythes et représentations de la femme, p. 57-66.

${ }^{26}$ Pseudonyme de Paul Devaux, auteur du roman Les Fellatores. Mours de la décadence, Paris, Union des bibliophiles, 1888. Un chapitre y est consacré au « rachildisme » (p. 201-207).

27 « Qui compose des livres tels, écrit un penseur éminent, ne s'appartient peutêtre pas plus qu'un homme double ; c'est un trop beau cas de tératologie. La tombe et l'oubli ne sont que pour le vulgaire. Lui il a les honneurs de la table de dissection et du musée de Dupuytren» ». M. Barrès, « Mademoiselle Baudelaire », annexe à Correspondance Rachilde-Barrès, p. 178. Il n'est pas anodin de préciser que le caractère des contacts des deux écrivains fut, pendant un certain temps, bien intime et Rachilde put se sentir profondément blessée par cette stigmatisation.

${ }^{28}$ Le roman, publié initialement à Bruxelles en 1884, parut chez Brossier en 1889.

${ }^{29}$ Erreur fréquemment répétée : en réalité, au moment d'écrire Monsieur Vénus, Rachilde avait vingt-quatre ans. 
expérience : Monsieur Vénus était pour lui une « autobiographie de la plus étrange des jeunes femmes $\gg^{30}$.

Dans les années à venir, l'élément de genre ne devait pas disparaître des commentaires des critiques. Il constitue le centre de l'article de Louis Dumur, écrit à l'occasion de la parution de L'Animale. Dumur découvre, sans doute dans la lignée de la pensée de Taine, un trait fondamental de l'écriture de Rachilde, à savoir la perversité. Il la présente comme une qualité, mais, somme toute, il finit par limiter le talent de la romancière à cet unique trait dans lequel il voit une spécificité féminine, en l'opposant au talent masculin, capable de transformer, d'aller vers l'idéalisation, inaccessible pour la femme, qui ne sait que déformer (on y retrouve la définition de la perversité proposée par Dumur) :

Mme Rachilde est restée femme, et elle fait de la littérature. Elle n'a point voulu, en abordant les lettres, se mettre à copier le modèle viril. Elle s'est donnée comme elle était : femme, et par conséquent perverse. Et elle est franchement, complètement, grandement, magnifiquement perverse. Elle ne simule ni la chasteté, ni la gauloiserie, ni la poésie, ni l'amour, ni la philosophie, ni la religion, ni aucune de ces choses créées par l'homme. Elle est seulement perverse. Elle a, enfin, apporté au monde le livre pervers. Nous l'attendions, qu'il soit le bienvenu ! ${ }^{31}$

Il semble, nous l'avons dit, que ce ton soit beaucoup plus typique de la première période où Rachilde apparait encore à plusieurs comme une « curiosité ». Plus tard, il est rare qu'on associe le caractère de son écriture à son sexe. Cependant, l'élément genré persiste et commence à fonctionner d'une manière quelque peu paradoxale. Les commentateurs plus tardifs de l'œuvre de Rachilde évoquent souvent le caractère mâle de son talent. Ce faisant, ils ne font que tomber dans les mêmes stéréotypes de genre, même si leurs jugements paraissent plus fondés. Ainsi, ils vantent l'originalité et la puissance de son imagination, sa plume « virile » et la placent, une fois de plus, en dehors du groupe d'écrivains-femmes. Remy de Gourmont, dans une analyse fortement imprégnée de misogynie ${ }^{32}$, conclue par ces mots :

30 M. Barrès, « Complications d'amour », préface à Monsieur Vénus, Paris, Brossier, 1889, annexe à Correspondance Rachilde-Barrès, p. 181-184.

31 L. Dumur, « Rachilde », La Plume, 15 mai 1893, p. 217-218.

32 Le texte s'ouvre par une longue analyse de l'incapacité organique de la femme de s'analyser où Remy de Gourmont déclare entre autres : « Avec une semblable nature il faudrait à une femme, pour se mettre au premier rang des hommes, un génie plus haut que le génie même des hommes les plus surélevés : c'est pourquoi si les œuvres marquantes des hommes sont assez souvent supérieures à l'homme, les œuvres les plus belles des femmes sont toujours inférieures à la valeur de la femme qui les a produites $\gg$. R. de Gourmont, « Rachilde », Le Livre des masques, Paris, Mercure de France, 1896, p. 187. 
Des pages comme la Panthère ou les Vendanges de Sodome montrent qu'une femme peut avoir des phases de virilité, écrire, à telle heure, sans le souci des coquetteries obligées ou des attitudes coutumières, faire de l'art avec rien qu'une idée et des mots, créer ${ }^{33}$.

Pour Adolphe Retté, « nerveuse, passionnée, virile dans le bon sens du mot, Rachilde n'évoque jamais le bas-bleu $»^{34}$. Jean Lombard parle de « cette curieuse femme de lettres, camarade de plume, dirons-nous volontiers $\gg^{35}$. D'autres vantent ses $\ll$ pages virilement et sincèrement écrites $»^{36}$, ou son $\ll$ style particulièrement mâle et nerveux $\gg^{37}$. En somme, restant fidèles à l'esprit misogyne de la fin de siècle, les critiques favorables à Rachilde croient nécessaire de la distinguer des autres écrivains femmes : d'après Henri Gausseron, « cet écrivain, pour être une femme, n'en est pas moins un violent $\gg^{38}$ et pour Henry-D. Davray elle est « une des rares femmes écrivains dont le style soit supportable, et même très séduisant $\gg^{39}$. Georges Casella l'exclut également du groupe de femmes auteurs : « Mme Rachilde est l'une des femmes de lettres que l'on oublie toujours lorsqu'on cite la liste nombreuse des écrivains féminins, romancières et poétesses. [... il y a dans les œuvres de Mme Rachilde un charme violent qui n'a rien des langueurs féminines et qui est la marque d'un maitre $\gg^{40}$. Enfin, Alfred Jarry met peut-être le doigt sur le problème : «Rachilde, l'auteur d'une trentaine de romans dont avoir écrit un seul suffirait à faire dire d'un homme qu'il a du génie... $\gg^{41}$

Que l'on l'accuse d'être une femme écrivain ou que l'on l'en défende, la question de son sexe revient systématiquement, et toujours de manière dépréciative. Rachilde ne peut que se percevoir comme membre d'une minorité peu favorisée. Aussi préfère-t-elle choisir son propre groupe d'exclusion.

\section{Bâtir sa force à partir de sa faiblesse}

Il faut reconnaitre que le fracas certain que fit la publication de Monsieur Vénus a permis de lancer la carrière de Rachilde. Les arguments ad personam que la critique avait abondamment utilisés n’ont pas été de peu dans ce succès.

33 Ibidem, p. 192. Les titres évoqués appartiennent au recueil Le Démon de l'absurde, publié au Mercure de France en 1894.

34 A. Retté, C. R. de La Tour d'amour, Le XIX siècle, 4 novembre 1899.

35 J. Lombard, La France Moderne, 5 mars 1891.

36 M. Du Plessys, Le Décadent, 15-31 mars 1889, p. 91.

37 M. Gauchez, La Société Nouvelle, août 1912, p. 203.

38 Revue Universelle, 1901, p. 181.

39 Le XIX ${ }^{e}$ siècle, 7 août 1912.

40 La Revue Illustrée, 5 janvier 1906.

41 A. Jarry, Albert Samain. Souvenirs, Paris, Victor Lemasle, 1907, p. 15. 
D'autre part, dès ce moment, la romancière était perçue comme, non seulement femme-écrivain, mais de plus femme excentrique, bizarre, perverse. Ses réactions à cette popularité fraîchement obtenue relèvent à la fois d'indignation et de satisfaction que son œuvre plaise aux lecteurs. Cette double attitude caractérisera l'écrivaine tout au long de sa carrière. Provocante, non seulement dans les choix des sujets de ses romans ${ }^{42}$, elle exploitera sa popularité ambiguë, tout en subissant le poids d'appartenir à une minorité. Dans certaines de ses préfaces, dans quelques insinuations de ses chroniques, on peut lire sa souffrance et son sentiment d'exclusion :

Et Rachilde aujourd'hui compte des ennemis dans cette vallée de larmes comme si elle n'était ni femme, ni jolie, ni jeune.

Tant pis pour elle ! jetée en pâture à l'extravagance, elle est devenue sa proie tout entière, elle doit être étrange ou ne plus être [...]

C'est que vivre est encore pour les monstres comme pour les autres la suprême joie ! 43

À d'autres occasions cependant, elle semble mieux préparée à assumer «son statut de romancière en même temps que celui de monstre $\gg$. Comme l'observe Nelly Sanchez, Rachilde a appris que cette position « lui permettait, mieux que n'importe quel rang social, de bousculer les conventions et jouir de libertés interdites à son sexe $\gg^{44}$. Il lui arrivait donc de mieux accepter les conséquences de sa réputation scandaleuse :

Je suis donc chien de lettres, à mon grand regret, hystérique de lettres, et si on pense que je ne mérite ni cet excès d'honneur ni cette indignité - il faut tout prévoir - je suis androgyne de lettres ${ }^{45}$.

De toute manière, cette forme d'isolement lui semblait préférable à celle d'être rangée dans la minorité féminine :

J'ai toujours regretté de ne pas être un homme, non point que je prise davantage l'autre moitié de l'humanité mais parce qu'obligée, par devoir ou par goût, de vivre

${ }^{42}$ Les titres de quelques-uns parlent d'eux-mêmes : La Virginité de Diane; Madame Adonis ; La Marquise de Sade ; L'Heure sexuelle; Les Hors nature ; La Tour d'amour ; L'Animale.

${ }^{43}$ Préface d’À Mort..., p. 174.

${ }^{44}$ N. Sanchez, « Rachilde ou la genèse (possible) de Monsieur Vénus », Nineteenth-Century French Studies 38, N 3 \& 4, Spring-Summer 2010, p. 262.

${ }^{45}$ Rachilde, préface à Madame Adonis, Paris, Monnier, p. XI-XII. 
comme un homme, de porter seule tout le plus lourd du fardeau de la vie pendant ma jeunesse, il eût été préférable d'en avoir au moins les privilèges sinon les apparences. Cette tendance à des allures masculines ne m’a nullement inspiré le désir de m'emparer de droits qui n'étaient pas les miens. J'ai toujours agi en individu ne songeant pas à fonder une société ou à bouleverser celle qui existait ${ }^{46}$.

Cette attitude n'est pas exempte de paradoxes : tout en se positionnant contre le féminisme, elle avoue cependant avoir été, « malgré moi, une des premières féministes de l'époque, sinon par le mérite, au moins par l'esprit révolutionnaire d'alors... qui est, à présent, l'esprit réactionnaire car ainsi tourne la roue du progrès mettant en bas ce qui fut en haut, sans, d'ailleurs, améliorer énormément l'existence $\gg^{47}$. Sa création reflète également ses dilemmes d'appartenance ou d'exclusion. Ses personnages sont, pour la plupart, des femmes, mais elles sont toutes des créatures exceptionnelles, androgynes, menant les hommes à leur guise, les dominant par l'intelligence, la force de caractère, et souvent, l'éducation. Raoule de Vénérande, de Monsieur Vénus, Mary Barbe, de La Marquise de Sade, Marcelle Désambres, de Madame Adonis, Éliante Donalger, de La Jongleuse, déplorent la décadence des mœurs actuelles et la faiblesse des hommes. « Dans l'antiquité, déclare Raoule, le vice était sacré parce qu'on était fort. Dans notre siècle, il est honteux parce qu'il nait de nos épuisements $\gg^{48}$. Mary Barbe attend ce « mâle effroyable qu'il lui fallait, à elle, femelle de la race des lionnes » et elle se désespère de cet affaiblissement général de la race française, elle-même « trop puissante pour devenir veule $\gg^{49}$. Éliante se fait de l'amour une religion, mais si elle veut se soumettre à un homme, elle sait bien ce qu'elle attend de lui : un sacrifice total et une sincérité parfaite. Ne pouvant pas trouver d'homme qui serait à la hauteur de ces exigences, elle préfère se tuer. Pareil pour Marcelle Désambres, un être exceptionnel qui doit périr face au triomphe évident de l'amour bourgeois. Il semble donc, en définitive, qu'une lecture psychologique des romans de Rachilde serait permise et que certains critiques, après tout, n'avaient pas une si mauvaise intuition...

\section{Conclusion}

Dès son enfance, Rachilde est consciente d'appartenir à une minorité. Son infirmité, son sexe féminin, ses ambitions littéraires font d'elle une exception. Mais ce qui, au début, constitue pour elle un handicap, devient, en fin des comptes, un

46 Rachilde, Pourquoi je ne suis pas féministe..., p. 6.

47 Ibidem, p. 8.

48 Rachilde, Monsieur Vénus..., p. 83.

49 Rachilde, La Marquise de Sade, Paris, Monnier, 1887, p. 373. 
atout. Au fil des années, elle revendique de plus en plus fermement sa position exceptionnelle. Elle choisit d'être une excentrique, une détraquée, un monstre. Elle ne se défend pas non plus devant l'étiquette de l'androgyne. Dans ses ouvrages, elle exploite toutes ces caractéristiques avec une étonnante régularité qui va au-delà des modes littéraires. En effet, en dépit de l'évolution évidente de son esthétique, dans laquelle le symbolisme remplaçait la décadence, pour ensuite faire place au roman d'aventure, ses personnages gardent toujours quelques traits communs avec le premier modèle, Raoule de Vénérande : suspendus entre les deux sexes, forts de leur intelligence et dominant les autres par leur personnalité. On peut, dès lors, se demander si une telle conséquence n'est pas révélatrice de sa vision toute particulière du problème de sexes. Bien avant les théories féministes des années 1990, notamment celles de Judith Butler, Rachilde ne pouvait peutêtre résoudre autrement ce qui s'imposait à elle dans sa propre vision du monde : sa différence par rapport à la majorité des femmes - et des hommes. Il ne faut pas s'en étonner, sachant à quel point les stéréotypes sur les femmes (et les hommes) continuent à exister au XXI ${ }^{\mathrm{e}}$ siècle. Les attitudes qu'elle endossait elle-même ou qu'elle décrivait dans ses romans, lui permettaient de s'adapter au style de vie accepté, tout en préservant son indépendance. Mais en même temps, elles lui barraient la route du féminisme.

\section{Rachilde, homme de lettres. Gender and exclusion}

At a time when the voice of feminists is getting stronger and the number of women writers is increasing, Rachilde's position seems ambiguous, if not paradoxical. A woman writer herself, an author of several novels appreciated by the public, a literary critic at the Mercure de France, she could become an important figure in the feminist movement. And yet she systematically flouts its claims; she also remains very critical of the women's writing, of which she refuses to be a part. Hence, it seems interesting to look for the causes of such an attitude. The scope of the subject and, even more, the contradictory statements of Rachilde herself, will at most allow us to formulate some hypotheses. However, we shall examine more closely the problem of Rachilde's belonging to one or more minorities.

Key words: Rachilde - feminism - minority - literary criticism

Mots-clés : Rachilde - féminisme - minorité - critique littéraire 